\title{
Prinzmetal Angina in Major Hemophilia a Patient: A Case Report
}

\author{
Romaric Mahutondji Massi ${ }^{1}$, Bienvenu Houssou ${ }^{1}$, Marième Camara ${ }^{1}$, Nisrine Khoubila ${ }^{2}$, \\ Asma Quessar ${ }^{2}$, Bouchra Oukkache ${ }^{1}$ \\ ${ }^{1}$ Departement of Biological Hematology of Ibn Rochd University Hospital, Casablanca, Morocco \\ ${ }^{2}$ Departement of Clinical Hematology and Pediatric Oncology of Ibn Rochd University Hospital, Casablanca, Morocco
}

Email address:

houbien85@yahoo.fr (Bienvenu H.)

To cite this article:

Romaric Mahutondji Massi, Bienvenu Houssou, Marième Camara, Nisrine Khoubila, Asma Quessar, Bouchra Oukkache. Prinzmetal Angina in Major Hemophilia a Patient: A Case Report. American Journal of Internal Medicine. Vol. 3, No. 4, 2015, pp. 194-196.

doi: 10.11648/j.ajim.20150304.17

\begin{abstract}
Introduction: Prinzmetal angina is a special type of acute coronary syndrome ST + wich correspond to a transient occlusion of a coronary vessel secondary to spasm. This type of acute coronary syndrome is very rare and is characterized by the presence of signs of myocardial ischemia on electrocardiogram but coronary angiography and coroscaner are frequently normals. Its management in hemophilia patients is difficult because of the use of anticoagulant and antiplatelet drugs wich increase bleeding risk. We report the case of a major hemophilia A patient which presented Prinzmetal angina. Observation : It is a 64 years old patient, hemophilia A major, chronic smoking (40 pack-year), not diabetic, not hypertensive, which had a retro sternal constrictive pain radiating to the shoulders. At admission he was consciousness. No breath in cardiac auscultation. The electrocardiography showed a heart rate at $61 \mathrm{bpm}$, the axis of the heart was normal. ST-segment elevation was noticed in DIII and AVF : ischemia in the cardiac lower area. Echocardiography was normal. The coroscaner was normal. The troponin I level was at $0.03 \mu \mathrm{g} / \mathrm{L}$ (Normal : 0-0.1 $\mu \mathrm{g} / \mathrm{L})$. This patient had a variant Prinzmetal angina. Treatement: Diltiazem Hydrochloride 60 mg 1 tablet / 8 hours. Acetylsalicylic acid $160 \mathrm{mg}$ IV and Clopidogrel $300 \mathrm{mg}$ IV the first day; relay with acetylsalicylic acid $100 \mathrm{mg}$ and clopidogrel $75 \mathrm{mg}$ per day. Transfusion of factor VIII at the dose of 40UI / Kg. Simvastatin $20 \mathrm{mg} 1$ tablet per day. Perindopril $5 \mathrm{mg} 1$ tablet the day. The evolution was favorable. Discussion and conclusion: Coronary syndromes are not frequent in morocco hemophilia patients. Their management is complex and involves the presence of an hematologist.
\end{abstract}

Keywords: Acute Coronary Syndrome, Hemophilia, Management

\section{Introduction}

The use of concentrated coagulation factors and the considerable reduction of the risk of infection by transfusion these last years, has improved the life expectancy of hemophilia patients from 30 to over 70 years. [1] Thus, haemophilia patients are now confronted to comorbidities that occur with age. The frequency of ischemic heart disease in hemophilia is increasing [2]. Kulkarni and al, in a cohort of 3,442 american haemophilia patients have shown that the prevalence of ischemic cardiovascular disease was $0.05 \%$ among haemophilia patients under 30 years old and over $15 \%$ in those over 60 years old [3]. Prinzmetal angina is a special type of acute coronary syndrome $\mathrm{ST}+$ wich correspond to a transient occlusion of a coronary vessel secondary to spasm. Coronary artery spasm has been recognized as an infrequent cause of acute coronary syndrome in patients with normal coronary angiography since 1959 by Prinzmetal et al [4]. The incidence of spontaneous coronary vasospasm has not been established, however, during coronary angiography, the incidence has been reported to be between $0.26 \%$ and $0.93 \%$ [5]. The incidence of Prinzmetal angina in hemophilia patients is not known.

The treatment of Prinzmetal angina like all acute coronary syndrome is based on the use of anticoagulants and antiplatelet drugs which increase the risk of bleeding. To minimize this risk, the factor deficiencie should be corrected. However, the administration of concentrates of coagulation factors increase the risk of cardiovascular ischemia. The precarious balance between bleeding and thrombosis should be considered while the treatment of an episode of ischemic heart disease in hemophilia patient begin. 
We report the case of a major hemophilia A patient who presented Prinzmetal angina.

\section{Observation}

It is a 64 years old patient, hemophilia A major, chronic smoking (40 pack-year), not diabetic, not hypertensive, which had a retro sternal constrictive pain radiating to the shoulders. He came to hospital 15 days after the bigining. At admission he was consciousness. The respiratory rate was at 20 cycles / minute ; blood preasure at 100/70 $\mathrm{mm} \mathrm{Hg}$ and heart rate at $60 \mathrm{bpm}$. No breath in cardiac auscultation. The electrocardiography showed a heart rate at $61 \mathrm{bpm}$, the axis of the heart was normal. ST-segment elevation was noticed in DIII and AVF : ischemia in the cardiac lower area. Echocardiography was normal. The coroscaner was normal. The troponin I level was at $0.03 \mu \mathrm{g} / \mathrm{L}$ (Normal : 0-0.1 $\mu \mathrm{g} / \mathrm{L}$ ). The hemostasis tests results were : Prothrombin time : 12.3 seconds (Normal 12-14 seconds), Partial thromboplastin time with activators : 98 seconds: (Normal : 24 seconds $+/-5$ ), fibrinogen : $4 \mathrm{~g} / \mathrm{L}$ (Normal : 2-4g/L). Factor VIII $<1 \%$. In addition this patient had a variant Prinzmetal angina. A staff was made between hematologists and cardiologists and decided the following treatement : Diltiazem Hydrochloride $60 \mathrm{mg} 1$ tablet / 8 hours. Acetylsalicylic acid $160 \mathrm{mg}$ IV and Clopidogrel $300 \mathrm{mg}$ IV the first day ; relay with acetylsalicylic acid $100 \mathrm{mg}$ and clopidogrel $75 \mathrm{mg}$ per day. Transfusion of factor VIII at the dose of $40 \mathrm{UI} / \mathrm{Kg}$, target: maintain the level of factor VIII more than $10 \%$. Simvastatin $20 \mathrm{mg} 1$ tablet per day. Perindopril $5 \mathrm{mg} 1$ tablet the day. The evolution was favorable. At Day 3 no pain and electrocardiogram was normal.

\section{Discussions}

Our case, the first case of variant angina in a hemophilia patient in the departement highlights the difficulties of the treatement of acute coronary syndrome in hemophilia patients. This treatment involves the use of anticoagulant and antiplatelets agents which increase the risk of bleeding in patients who constitutionally present a high risk of bleeding.

Severe coronary artery spasm is a common occurrence in patients with endothelial dysfunction with and without atherosclerotic coronary artery disease. It plays an important role in the pathogenesis of angina pectoris, Prizmetal's variant angina, and acute myocardial infarction. The primary cause of coronary vasospasm is focal hyperreactivity that is commonly due to a deficiency in endothelial nitric oxide (NO), which is frequently caused by hyperlipidemia and coronary atherosclerosis [6]. NO is the major vasodilator chemical produced by the coronary endothelium and produces coronary dilation by increasing cyclic guanosine monophosphate in vascular smooth muscle cells. It is continuously released from the coronary endothelium under resting conditions, contributing to the patency of the coronary artery and is an important determinant of dilation during increases in demand (i.e., exercise) [7]. The pulsatile arterial blood flow is the primary determinant of NO release from the coronary endothelium. The rhythmic changes in vessel diameter and deformation of the arterial wall induce transient increases in intracellular calcium in the endothelium. Increased endothelial cell calcium activates NO synthetase, which induces synthesis of $\mathrm{NO}$, leading to coronary dilation. Nitroglycerin, which is converted into NO, and calcium channel blockers, which decrease calcium entry into vascular muscle cells, facilitate vascular relaxation, and are usually beneficial in the treatment of recurrent coronary spasm.

The treatment of acute coronary syndrome is well codified in subjects without hemostasis disorder and the European Society of Cardiology recommandations of 2007 and 2008 in all cases involve reperfusion (intravenous thrombolysis or primary coronary angioplasty) associate with medical treatment which respect the acronym BASIC (Beta-blocker, Aspirin, Statin, ACE inhibitor, correction of cardiology risk factors) $[8,9]$.

Antiplatelet agents have an important place in this treatment. Aspirin is administered at an intravenous dose of 160 to $325 \mathrm{mg}$ followed by a dose of 75 to $100 \mathrm{mg}$. Clopidogrel is recommended in combination with aspirin. It must be administered at the dose of $300 \mathrm{mg}$ followed by a maintenance dose of $75 \mathrm{mg}$ for 12 months. In addition to antiplatelet therapy anticoagulant therapy (Fondaparinux, low molecular weight heparins (LMWH), unfractionated heparin) is recommended for acute coronary syndrome. The duration of anticoagulation is variable, it can be stopped 24 hours after invasive procedures. In conservative strategies, anticoagulant treatment can be continued and stopped as soon as the patient leave hospital [2].

The application of this treatment is a real dilemma in haemophilia patient because firstly anticoagulants and antiplatelet treatment increase the risk of bleeding, secondly the correction of coagulation factor deficiency increase the risk of thrombosis.

The European Society of Cardiology in its guidelines published in 2013, proposes that a replacement therapy is necessary before the start of anticoagulant treatment and anti platelet combination therapy that can not be started until the level of the coagulation factor is under $5-15 \%$. The duration of this treatment should be a minimum [10]. The usually attitude in practice are the following:

-Acute coronary syndrome in a haemophilia with factor levels below 10\%: Start immediately clopidogrel and aspirin, and do the injection of antihemophilic factor (40 IU / kg (FVIII) or $80 \mathrm{IU} / \mathrm{kg}$ (FIX)). Then start heparin at anticoagulation dose and do coronary angiography. Control the coagulation factor levels every 12 hours. A new administration of anti hemophilic factor is made according to the results.

- Acute coronary syndrome in a haemophilia with factor levels above 10\%: Start immediately clopidogrel, aspirin and heparin at iso anticoagulation dose, do the injecting of anti-hemophilic factor (40 IU / kg (FVIII) or $80 \mathrm{IU} / \mathrm{Kg}$ (FIX)). Then, adminstrate heparin at anticoagulation dose and do coronary angiography. 
Control the levels of coagulation factor every 12 hours. A new administration of anti hemophilic factor is made according to the results.

- In all cases, the systematically use of beta blocker, ACE inhibitor and statin is recommand.

- In the particular case of variant Prinzmetal angina the calcium blocker wich have vasodilator property is recommand.

In our case, according to the level of factor VIII less than $1 \%$ and the risk of bleeding associated with the use of heparin we opt for not to do coronary angiography and we chose the realisation of coroscaner in first time until this attitude is not recommand. It is because of the same reasons (high bleeding risk according to factor VIII less than 1\%) that we choose the medical treatment option and avoid surgery which need the use of anticoagulation whith heparin and increase the bleeding risk. Fogarty PF and al had founded in a recent review on the management of 19 hemophilia patients who had acute coronary syndromes that none of eight patients with severe haemophilia underwent Coronary Artery Bypass Grafting (CABG). They had founded also that in $25 \%$ of cases the initial acute coronary syndrom management protocol was altered because of blinding disorder [11]. We choose to administrate factor VIII for our patient because there are no specific evidence - based guidelines for patients with coagulation defects according antithrombotic treatement. Therefore, Mannucci PM recommanded that replacement therapy should be use to control the incrased risk of bleeding inherent in the use of antiplatelet and anticoagulant drugs [12]. In coronary spasm, long- acting nitrate and calcium channel blockers a considered as first-line therapies. Usually, a calcium channel blocker in a moderate to hight dose is stated; patients with very active disease can require a combination combination of nitrates and two calcium channels blockers of differentes classes [13]. In our case, we use only calcium channel blocker at moderate dose and the evolution was good.

\section{Conclusion}

The management of acute coronary syndrome in a haemophilia is difficult and requires the presence of a hematologist. The itterative administration of coagulation factor in the treatment requires the research of antibodies anti coagulation factor at the begining of the protocol.

\section{References}

[1] Darby SC, Wan Kan S, Spooner RJ et al. Mortality rates, life expectancy, and causes of death in people with hemophilia A or B in the United Kingdom who where not infected with HIV. Blood 2007;110:815-25.

[2] Cayla G, Morange PE, Chambost H, Schved JF. Hémophilie et pathologies cardiovasculaires. AMC pratique. Hors-série 1. Juin $2010: 1-3$

[3] Kulkarni R, Soucie JM, Evatt BL, Hemophilia Surveillance System Project Investigators. Prevalence and risk factors for heart disease among males with hemophilia. Am J Hematol 2005;79:36-42.

[4] Prinzmetal M, Kennamer R, Merliss R, et al. Angina pectoris. A variant form of angina pectoris; preliminary report. Am J Med. 1959;27:375e388.

[5] Chahine RA, Raizner AE, Ishimori T, et al. The incidence and clinical implications of coronary artery spasm. Circulation. 1975;52:972e978.

[6] Feliciano L, Henning R. Coronary artery blood flow: physiologic and pathophysiologic regulation. Clin Cardiol 1999;22:775 - 86 .

[7] -Kelm M, Schrader J. Control of coronary vascular tone by nitric oxide. Circ Res 1990;66:1561 - 75.

[8] Bassand JP, Hamm CW, Ardissino D et al. Guidelines for the diagnosis and treatment of non-ST-segment elevation acute coronary syndromes. Eur Heart J. 2007;28:1598-660.

[9] Van de Werf F, Bax J, Betriu A et al. Management of acute myocardial infarction in patients presenting with persistent ST-segment elevation: the Task Force on the Management of ST-Segment Elevation Acute Myocardial Infarction of the European Society of Cardiology. Eur Heart J 2008;29:2909-45.

[10] Staritz P, de Moerloose P, Schutgens R, Dolan G. Applicability of the European Society of Cardiology guidelines on management of acute coronary syndromes to people with haemophilia - an assessment by the ADVANCE Working Group. Haemophilia. 2013 Nov;19(6):833-40.

[11] Fogarty PF, Mancuso ME, Kasthuri R, Bidlingmaier C, Chitlur M, Gomez K, et al. Presentation and management of acute coronary syndromes among adult persons with haemophilia: result of an international, retrospective, 10-years survey. Haemophilia. 2015 Feb 17. Doi: 10. 1111/hae.12652.

[12] Mannucci PM. Management of antithrombotic therapy for acute coronary syndromes and atrial fibrillation in patients with hemophilia. Expert Opin Pharmacother 2012;13: 505-10.

[13] Anderson JL, Adams CD, Antman ME, Bridges CH, Califf RM, Donald E, et al. 2012 ACCF/AHA 2007 guidelines for the management of patients with unstable angina/non-STelevation myocardial infarction: a report of the American College of Cardiology Foundation/American Heart Association Task Force on Practice Guidelines. Circulation 2013; 127: e663-828. 